\title{
Relationship between Serum Aprindine Concentration and Neurologic Side Effects in Japanese
}

\author{
Yoshimasa Tsuchishita, ${ }^{a}$ Kyoko Fukumoto, ${ }^{b}$ Masaaki Kusumoto, ${ }^{a}$ and Kazuyuki Ueno*, $b$ \\ ${ }^{a}$ Department of Pharmacy, Maizuru Kyosai Hospital, Federation of National Public Services and Affiliated Mutual \\ Associations; 1035 Hama, Maizuru, Kyoto 625-8585, Japan: and ${ }^{b}$ Department of Pharmaceutical Sciences, Niigata \\ University of Pharmacy and Applied Life Sciences; 265-1 Higashijima, Akiha-ku, Niigata 956-8603, Japan. \\ Received November 26, 2008; accepted January 8, 2009
}

\begin{abstract}
The aim of this study was to evaluate the relationship between the neurologic side effects associated with serum aprindine concentrations and the safety range of aprindine for the prevention of neurologic side effects in 142 Japanese inpatients. Serum aprindine concentrations were determined by high-performance liquid chromatography. A poor positive correlation was observed between dose and serum aprindine concentration $\left(r^{2}=0.0419, p=0.0114\right)$, and between age and ratio of serum aprindine concentration to the dose per body weight of aprindine $\left(r^{2}=0.0159, p=0.121\right)$. When aprindine concentration was $<1 \mu \mathrm{g} / \mathrm{ml}$, almost no patients showed neurologic side effects associated with aprindine. On the other hand, about $50 \%$ of the patients showed neurologic side effects when aprindine concentrations were $>1 \mu \mathrm{g} / \mathrm{ml}$. Here, the side effects associated with aprindine such as dizziness or intention tremors were observed in 15 patients, which later disappeared after discontinuance of aprindine therapy or a decrease in the dose. In conclusion, serum aprindine concentration should be maintained under approximately $1 \mu \mathrm{g} / \mathrm{ml}$ in Japanese patients to prevent neurologic side effects.
\end{abstract}

Key words aprindine; neurologic side effect; therapeutic drug monitoring; concentration

Aprindine is a class I antiarrhythmic agent widely used clinically for the treatment and prophylaxis of supraventricular and ventricular arrhythmias. ${ }^{1-3)}$ Oral administration of aprindine does not decrease heart contractility, ${ }^{4}$ and therefore it is recommended as the treatment of choice to prevent symptomatic atrial fibrillation in patients with cardiac dysfunction. ${ }^{1)}$ The antiarrhythmic effect of aprindine is proportional to its serum concentration, and the effective therapeutic ranges may be from 1 to $2 \mu \mathrm{g} / \mathrm{ml}$ in the U.S. and Europe, $\left.{ }^{5,6}\right)$ and from 0.25 to $1.25 \mu \mathrm{g} / \mathrm{ml}$ in Japan. ${ }^{7)}$ The variation may be related to racial difference in sensitivity to aprindine. More specifically, the pharmacokinetics of aprindine is nonlinear, ${ }^{7,8)}$ and it is metabolized exclusively by the liver primarily catalyzed by CYP2D6. ${ }^{9)}$ It is known that the incidence rate of poor metabolizers of CYP2D6 substrates is within 5-10\% among Americans and Europeans and about $0.5 \%$ in Japanese. Intermediator and ultra-rapid metabolizers of CYP2D6 are included in the same group as extensive metabolizers. ${ }^{10,11)}$ Therefore, drugs catalyzed by CYP2D6 have large interindividual variation.

Previous studies have suggested that aprindine produces neurologic side effects such as dizziness and intention tremors when plasma levels are continuously maintained at $2 \mu \mathrm{g} / \mathrm{ml}$ in the U.S. ${ }^{12)}$ However, while there are several case reports on aprindine-induced neurologic side effects in clinical studies, no reports thus far have suggested its safety range for the prevention of aprindine-associated neurologic side effects in Japanese patients.

The aim of this study was to evaluate the relationship between the neurologic side effects of aprindine and its serum concentration, as well as the safety range of aprindine for the prevention of neurologic side effects in Japanese patients.

\section{MATERIALS AND METHODS}

Materials Aprindine and amitriptyline were provided by Bayer Pharmaceutical Co., Ltd. (Osaka, Japan) and Wako
Pure Chemical Industris Co., Ltd. (Osaka, Japan), respectively. To administer to patients, commercial Aspenon ${ }^{\circledR} 10$ capsules (containing $10 \mathrm{mg}$ of aprindine, Bayer Pharmaceutical Co., Ltd., Osaka, Japan) were used. Acetonitrile used in high-performance liquid chromatography (HPLC) was of reagent grade, as well as the other reagents which were used without further purification.

Subjects Clinical laboratory and pharmacokinetic data were collected from 142 Japanese inpatients (age: mean \pm S.D., $69.8 \pm 8.2$ year, range, $40-85$ year; body weight: mean \pm S.D., $47.6 \pm 9.4 \mathrm{~kg}$, range, $27-99 \mathrm{~kg}$ ) who received maintenance aprindine therapy (tid, 7:00, 12:00 and 19:00) as determined by their cardiologists for the treatment of atrial fibrillation. The subjects were hospitalized at Maizuru Kyosai Hospital (Kyoto, Japan) between September 1998 and July 2008 , and data were collected retrospectively. Side effects including neurologic, gastrointestinal and cardiovascular side effects, etc., were monitored by cardiologists, nurses and pharmacists, and the side effects were recorded in the case history sheets. Because all the subjects were inpatients, compliance was ensured by a nurse or pharmacist. Patients were excluded if they were being administered rifampin, phenobarbital, quinidine, cimetidine, or other drugs known to affect the pharmacokinetics of aprindine, antidepressants and antiepileptic agents. No subjects developed hepatic dysfunction (i.e., ALT or AST level $>100 \mathrm{IU} / 1$ during this study. Written informed consent was obtained from all the subjects before enrollment. The study was approved by the Ethics Committee of Maizuru Kyosai Hospital, Federation of National Public Services and Affiliated Mutual Associations and Niigata University of Pharmacy and Applied Life Sciences.

Blood Sampling Blood samples (about $5 \mathrm{ml}$ ) were drawn at 06:00 from an arm vein and serum was obtained by centrifugation (3000 rpm for $10 \mathrm{~min}$, room temperature). Both aprindine concentrations and clinical laboratory values were measured using the same serum. The side effects were moni- 
tored on the day of blood sampling of serum aprindine concentrations. When the side effects were found in case where no blood samples were drawn, blood sample was drawn at the next morning.

Assay Serum aprindine concentrations were determined by HPLC with amitriptyline as an internal standard (IS). Briefly, $1 \mathrm{ml}$ of serum, $20 \mu \mathrm{l}$ of amitriptyline $(100 \mu \mathrm{g} / \mathrm{ml}), 500$ $\mu \mathrm{l}$ of $0.2 \mathrm{M}$ sodium carbonate, and $6 \mathrm{ml}$ of hexane were vortexed for $30 \mathrm{~s}$. After that, aprindine was extracted with hexan followed by hexan evaporation. The residue was then reconstituted in the mobile phase before injection into the HPLC system, which consisted of a reverse-phase column (ShimPack, CLC-ODS, length and inter-width of the column: $25 \mathrm{~mm}$ and $6 \mathrm{~mm}$. Shimadzu Corp., Kyoto, Japan) and an ultraviolet absorbance detector set at $259 \mathrm{~nm}$. The mobile phase consisted of a mixture of $0.1 \mathrm{~m}$ phosphate buffer $(\mathrm{pH}$ $3.5)$ and acetonitrile ( $65: 35$ by volume), and the flow rate was $1.0 \mathrm{ml} / \mathrm{min}$ the retention times of IS and aprindine were 12 and $20 \mathrm{~min}$, respectively. The minimum measurable concentration of this system was $50 \mathrm{ng} / \mathrm{ml}$ in $1.0 \mathrm{ml}$ serum. Interand intra-day variations were $<5.0 \%$.

Data Analysis Because all subjects were given fixed maintenance doses of aprindine for at least 2 weeks, it was assumed that most of their serum concentrations had reached a steady state. All blood samplings were performed at the same time of the day (i.e., 06:00). Therefore, to evaluate the pharmacokinetics of this compound, the ratio of serum aprindine concentration to the dose per body weight (C/D) was used in this study.

Statistical Analysis Data are expressed as mean \pm standard deviation (S.D.). Statistical analysis was performed using regression analysis and Student's $t$-test and $\chi^{2}$-test. Statistical significance was set at $p<0.001$.

\section{RESULTS}

Figure 1 shows the relationship between dose $(\mathrm{mg} / \mathrm{kg})$ and serum aprindine concentration in the groups with (solid triangles) and without (open circles) neurologic side effects. A poor positive correlation was observed between dose and serum aprindine concentration $\left(r^{2}=0.0419, p=0.0114\right)$. The relationship between age and the $\mathrm{C} / \mathrm{D}$ of aprindine in the groups with (solid circles) and without (open circles) nerurologic side effects is shown in Fig. 2. Mean \pm S.D. of ages in the group with and without neurologic side effects were $67.1 \pm 8.2$ and $70.1 \pm 8.1$ year. No significant correlation was observed between age and the C/D of aprindine $\left(r^{2}=0.0159\right.$, $p=0.121)$. In order to evaluate the frequency of aprindine-associated neurologic side effects as a function of the serum trough concentration of aprindine, the subjects were divided into 5 groups according to concentration, namely, in the range of (1) $<0.75$, (2) from 0.75 to 1.00 , (3) from 1.00 to 1.25 , (2) from 1.25 to 1.50 , and (5) $>1.50 \mu \mathrm{g} / \mathrm{ml}$. The results are shown in Fig. 3. When the concentrations were $<1 \mu \mathrm{g} /$ $\mathrm{ml}$, almost no patients showed neurologic side effects associated with aprindine. The age of 2 patients, who showed neurologic side effects within their concentration were $<1 \mu \mathrm{g} / \mathrm{ml}$ was 82 and 68 years old, respectively. On the other hand, about $50 \%$ of the patients showed neurologic side effects when aprindine concentrations were $>1 \mu \mathrm{g} / \mathrm{ml}$. A significant difference was observed in the frequency of the appearance

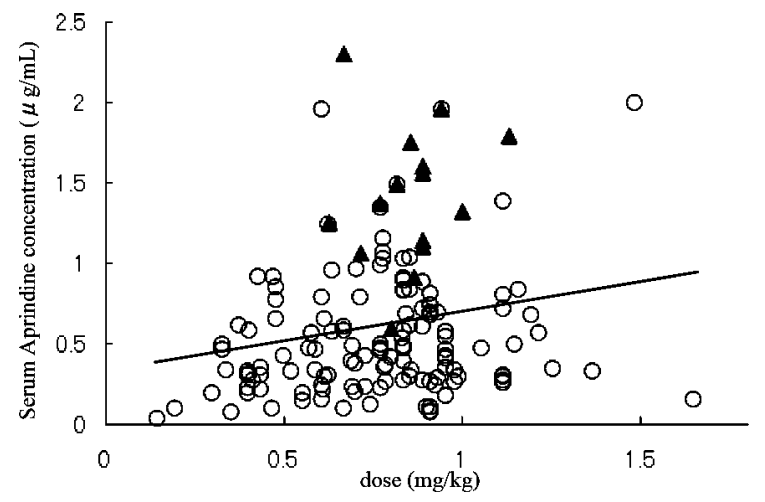

Fig. 1. Relationship between Aprindine Dose (mg/kg) and Its Serum Concentration in Groups with (Solid Triangles) and without (Open Circles) Neurologic Side Effects

Symbols represent individual data. The regression equation method is determined by $y=0.376 x+0.333, r^{2}=0.0419, p=0.0114$, as shown with a solid line.

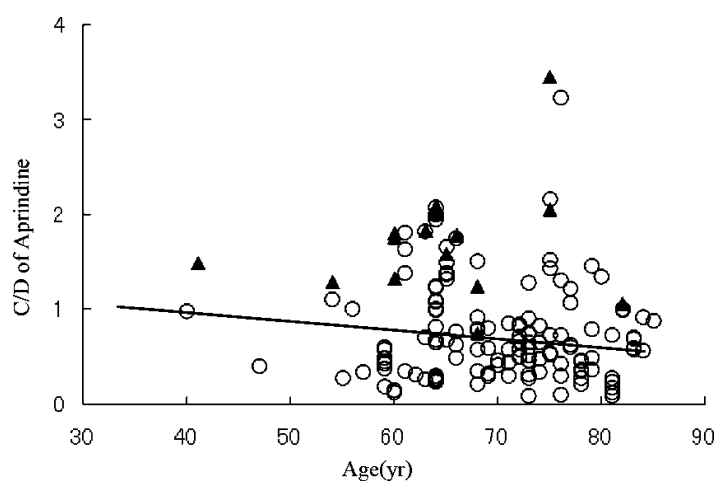

Fig. 2. Relationship between Age and C/D of Aprindine in Groups with (Solid Triangles) and without (Open Circles) Neurologic Side Effects

Symbols represent individual data. The regression equation method is determined by $y=-0.0089 x+1.46, r^{2}=0.0159, p=0.121$, as shown with a solid line.

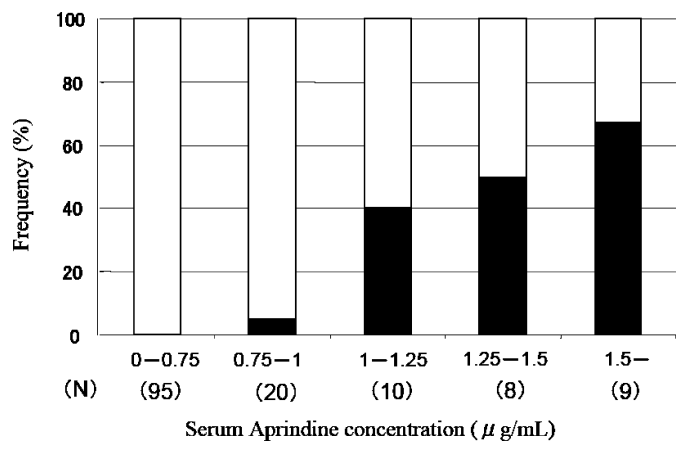

Fig. 3. Frequency of Central Nervous Side Effects Associated with Aprindine Grouped by Concentration

Open and closed columns denote without and with side effects, respectively.

of side effects between the 2 groups (a concentration of $<1$ $\mu \mathrm{g} / \mathrm{ml}$ versus $>1 \mu \mathrm{g} / \mathrm{ml}$ ). Here, aprindine-associated side effects such as dizziness or intention tremors were observed in 15 patients. The side effects disappeared with the discontinuance of aprindine therapy in 5 subjects and by decreasing aprindine dose in 10 subjects, whose serum concentrations at the first morning (06:00) after the side effects disappeared were $<1 \mu \mathrm{g} / \mathrm{ml}$ (Fig. 4). 


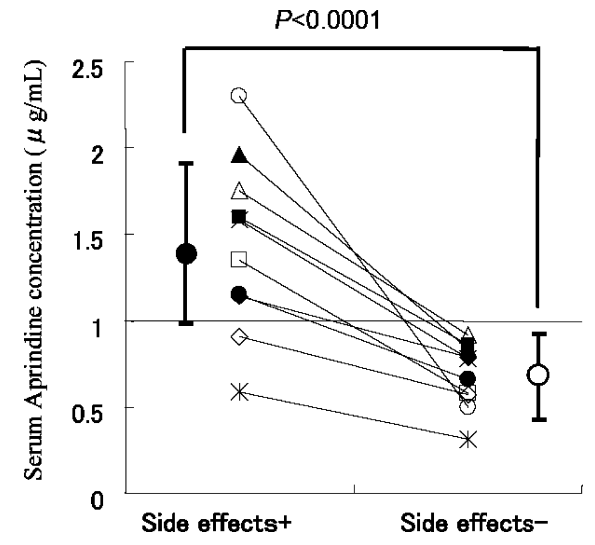

Fig. 4. Comparison of Serum Aprindine Concentrations between with or without Neurologic Side Effects in 10 Patients

\section{DISCUSSION}

Aprindine is a class I antiarrhythmic agent widely used clinically for the treatment and prophylaxis of supraventricular and ventricular arrhythmias. ${ }^{1-3)}$ The pharmacokinetics of aprindine is nonlinear and it is exclusively metabolized by the liver, primarily catalyzed by CYP2D6. ${ }^{9}$ It is known that drugs catalyzed by CYP2D6 have large interindividual variation. ${ }^{10,11,13)}$ As shown in Fig. 1, a poor positive correlation was observed between dose $(\mathrm{mg} / \mathrm{kg})$ and serum aprindine concentration, and it was tendency that the pharmacokinetics of apindine were nonlinear within the therapeutic range of serum concentrations. Therefore, the determination of aprindine serum concentration and appropriate dosage adjustments are needed to optimize aprindine therapy. Aprindine has a relatively narrow therapeutic window, and previous studies have suggested that it produces neurologic side effects such as dizziness and intention tremors when its plasma levels are continuously maintained at $2 \mu \mathrm{g} / \mathrm{ml}$ in the U.S. ${ }^{12)}$ Here, aprindine-associated side effects were observed in 15 of 142 patients, where aprindine concentrations were $<2 \mu \mathrm{g} /$ $\mathrm{ml}$. The antiarrhythmic effect of aprindine is proportional to its serum concentration, and the effective therapeutic ranges may be from 1 to $2 \mu \mathrm{g} / \mathrm{ml}$ in the U.S. and Europe, and from 0.25 to $1.25 \mu \mathrm{g} / \mathrm{ml}$ in Japan. ${ }^{7)}$ Therefore, it is suggested that not only the antiarrhythmic effect but also the neurologic effect of aprindine shows racial differences in sensitivity between Japanese and other populations.

As stated in the package insert of Aspenon ${ }^{\circledR}$, the side effects of aprindine must be monitored when it is administered to elderly patients. However, as shown in Fig. 2, no correlation was observed between age and the $\mathrm{C} / \mathrm{D}$ of aprindine, and side effects were observed in both young and elderly patients. No siginificant difference was observed in age between in the group with and that without the neurologic side effects. However, there exists a comparatively large effect of age on the pharmacokinetics of the drug, which is mainly excreted in the urine. Aprindine is mainly metabolized exclu- sively by the liver. The results therefore suggest that serum aprindine concentration should be monitored not only in elderly patients but also in young patients for the prevention of neurologic side effects.

On the other hand, as shown in Fig. 3, about half of the subjects showed neurologic side effects, whose aprindine concentrations were $>1 \mu \mathrm{g} / \mathrm{ml}$. The frequency of the appearance of aprindine-associated side effects observed in 15 patients increased with serum aprindine concentration. However, the side effects disappeared after discontinuing aprindine therapy or decreasing the dose. At the time of the appearance of side effects, the serum aprindine concentrations were about $1 \mu \mathrm{g} / \mathrm{ml}$ (Fig. 4). A few reports regarding as safety range and side-effects of aprindine had been published, and although there are several case reports on aprindine-induced neurologic side effects in clinical studies, ${ }^{12}$ no reports have thus far suggested its safety range for the prevention of neurologic side effects in Japanese patients. Our findings suggest that aprindine side effects are related to its serum concentration rather than age. These data suggest that serum aprindine trough concentrations should be maintained under approximately $1 \mu \mathrm{g} / \mathrm{ml}$ to prevent neurologic side effects associated with aprindine therapy, and it is needed to evaluate the safety range with a large population.

In conclusion, in order to prevent aprindine-associated neurologic side effects, serum aprindine concentration should be maintained under approximately $1 \mu \mathrm{g} / \mathrm{ml}$, and it is needed to monitor the serum concentration because the pharmacokinetics of aprindine is nonlinear.

\section{REFERENCES}

1) Kodama I., Ogawa S., Inoue H., Kasanuki H., Kato T., Mitamura H., Hiraoka M., Sugimoto T., Jpn. Circ. J., 63, 1-12 (1999).

2) Fasola A. F., Noble R. J., Zipes D. P., Am. J. Cardiol., 39, 903-909 (1977).

3) Kesteloot H., Van Mieghem W., De Geest H., Acta Cardilol., 28, $145-165$ (1973).

4) Simada R., Araki H., Orita Y., Nakagaki O., Nakamura M., Excerpta Med., 5, 348-356 (1983).

5) Vlay S. C., Kallman C., Reid S. P., J. Am. Coll. Cardiol., 5, 738-743 (1985).

6) Van Durme J. P., Bogert M. G., Rosseel M. T., Eur. J. Clin. Pharmacol., 7, 343-346 (1974).

7) Yokota M., Inagaki H., Uematsu H., Enomoto N., Goto J., Sotobata I., Takahashi A., Arzneim. Forsch.-Drug Res., 37, 184-188 (1987).

8) Kobari T., Itoh T., Hirakawa T., Namekawa H., Suzuki T., Satoh T., Iida N., Ohtsu F., Hayakawa H., Eur. J. Pharmacol., 26, 129-131 (1984).

9) Ebner T., Eichelbaum M., Br. J. Clin. Pharmacol., 35, 426-430 (1993).

10) Nakamura K., Goto F., Ray W. A., McAllister C. B., Jacqz E., Wilkinson G. R., Branch R. A., Clin. Pharmocol. Ther., 38, 402-408 (1985).

11) Horai Y., Taga J., Ishizaki T., Ishikawa K., Br. J. Clin. Pharmacol., 29, $111-115$ (1990).

12) Fasola A. F., Charmichael R., Acta Cardiol., 18, 317-333 (1974).

13) Shimizu M., Ohota T., Kishida M., Ogata H., Drug Metab. Pharmacokinet., 17, 292-299 (2002). 